\title{
Variations in the Branching Pattern of the Aortic Arch Detected with Computerized Tomography Angiography
}

\author{
Pasaoglu Lale, Ugur Toprak, Gökhan Yagız, Tunca Kaya, and Sadık Ahmet Uyanık \\ Department of Radiology, Ankara Numune Training and Research Hospital, Ulku Mahallesi Talatpasa Bulvari No. 5, \\ Altindag, 06100 Ankara, Turkey \\ Correspondence should be addressed to Gökhan Yagız; ygzgkhn@yahoo.com
}

Received 8 March 2014; Accepted 4 June 2014; Published 29 June 2014

Academic Editor: Letterio S. Politi

Copyright (C) 2014 Pasaoglu Lale et al. This is an open access article distributed under the Creative Commons Attribution License, which permits unrestricted use, distribution, and reproduction in any medium, provided the original work is properly cited.

Objectives. The aim was to determine the prevalence of aortic arch variations in 881 patients who underwent neck CT angiography for various reasons. Material and Methods. 881 patients were included in the study who had undergone neck CT angiography between 2010 and 2013. Results. Of 881 patients, 770 (87.4\%) patients with classic branching pattern of the aortic arch (AA) were observed. Variations in branching pattern were seen in $111(12.6 \%)$ patients. The most common variation was the origination of the left common carotid artery (LCCA) from the brachiocephalic trunk (BCT). This pattern was observed in 64 (7.2\%) cases. In 25 $(2.8 \%)$ cases, the left vertebral artery (LVA) originated directly from the AA between the origin of the LCCA and left subclavian artery (SCA). 17 (1.9\%) cases had aberrant right subclavian artery. Three (0.3\%) cases showed right aortic arch. Two cases had right aortic arch with aberrant left subclavian artery. Conclusions. Variations in the branching pattern of the AA are not rare. Head and neck surgeons and interventional radiologists should be aware of aortic arch variations. CTA is a reliable imaging method for demonstrating anatomical features and variations of the AA.

\section{Introduction}

In the classical anatomical configuration, the aortic arch (AA) is left sided and the most common branching pattern of the AA comprises of three great vessels; first the brachiocephalic trunk (BCT), then the left common carotid artery (LCCA), and finally the left subclavian artery (SCA) from right to left. The BCT branches into right SCA and right common carotid artery (RCCA). This branching pattern occurs in 64.9-94.3\% of the cases and it is described as "normal" [1-6]. Variations in the branching pattern of the AA range from differences in the origins of different branches to the number of branches $[2,4]$. Development of the aorta takes place during the third week of gestation [5]. Six pairs of aortic arches, the so-called branchial arch arteries, develop between the ventral and dorsal aortae. These variations of the AA can be explained by persistence of segments of the aortic arches that normally regress or disappearance of segments that normally remain, or both $[1,5,6]$.
Anatomical variations in the branching pattern of AA are significant for diagnostic, surgical, and interventional procedures of the thorax and neck. The purpose of this study is to review the computed tomography angiography (CTA) appearance of anatomical variations in branching pattern and position alterations of the $\mathrm{AA}$ and to determine the prevalence in 881 patients.

\section{Material and Methods}

The approval for this retrospective study was obtained from the institutional review board. 881 consecutive cases that underwent carotid CTA examination for different purposes were examined retrospectively. These CTA examinations belonged to $527(\% 59.8)$ male and 354 (\%40.2) female patients and they were performed during a 3-year period, from 2010 to 2013. The age range was $19-93$ years. Mean age of all the cases was 62 (19-93), mean age of males was 59 (21-93), and mean 
age of females was 62 (19-91). There was not any significant age difference between male and female patients $(P>0.05)$.

2.1. CTA Protocol. CTA examinations were performed using a 16-detector and 64-detector scanner (Aquilion 16, Aquilion 64, Toshiba Medical Systems, Otawara, Japan). In our standard CTA protocol for brain and neck examinations, a scan area from the aortic arch to the vertex level in supine position was adopted as a field of view. During examination, an 18-20 gauge angiocath in the antecubital vein was used to inject $90-120 \mathrm{~mL}$ of nonionic iodinated contrast media using bolus-tracking method with an automatic injector at a rate of $5 \mathrm{~mL} / \mathrm{sec}$ and $40 \mathrm{~mL}$ of saline solution at a rate of $4 \mathrm{~mL} / \mathrm{sec}$, respectively. The region of interest was positioned at the aortic arch, and the threshold was set to $130 \mathrm{HU}$. When the threshold was surpassed, helical scanning was automatically initiated. Scan parameters were $120 \mathrm{kV}, 300 \mathrm{~mA}$, and $420 \mathrm{msec}$ rotation time with a slice thickness of $1 \mathrm{~mm}$ and increments of $0.5 \mathrm{~mm}$, using a detector collimation of $16 \times 1 \mathrm{~mm}$.

2.2. Image Analysis. The obtained axial images from CTA examinations were transferred to the work station (Vitrea 2, Vital Images Inc., Minnetonka, MN, US) for analysis. Axial source data images, multiplanar reformat images, and postprocessed (maximum intensity projection and 3D volume rendered) images were evaluated. Image analysis was performed by a radiologist experienced in CTA and all the examinations were reviewed by the same radiologist.

2.3. Statistical Analysis. Data analysis was carried out using commercially available software (statistical package for social sciences, SPSS Inc., Chicago, Illinois, US). Mean minimum and maximum values of participants' demographic data were performed.

\section{Results}

Eight hundred and eighty-one cases (527 males and 354 females, age range 19-93 years) were included in this study.

In $770(87.4 \%)$ cases (516 males and 354 females) the classic branching pattern of the AA was observed. This pattern which is described as "normal" in the literature gives rise to three branches: BCT, CCA, and left SCA from right to left (Figure 1).

$111(12.6 \%)$ cases (65 males, 46 females) showed variations in branching pattern. The most common variation was origination of the LCCA from the BCT (arch with two branches, BCT with LCCA, and left SCA). This pattern was observed in $64(7.2 \%)$ cases (43 males, and 21 females) (Figure 2).

In 25 (2.8\%) (14 males, 11 females) cases, the left vertebral artery (LVA) originated directly from the AA between the origin of the LCCA and left SCA, providing an AA pattern with four branches (BCT, LCCA, LVA, and left SCA from right to left) (Figure 3).

17 (1.9\%) (6 males, 11 females) cases had aberrant right subclavian artery (RCCA, LCCA, left SCA, and right SCA). The right SCA was the last branch of the AA coursing to the right behind the esophagus (Figure 4).

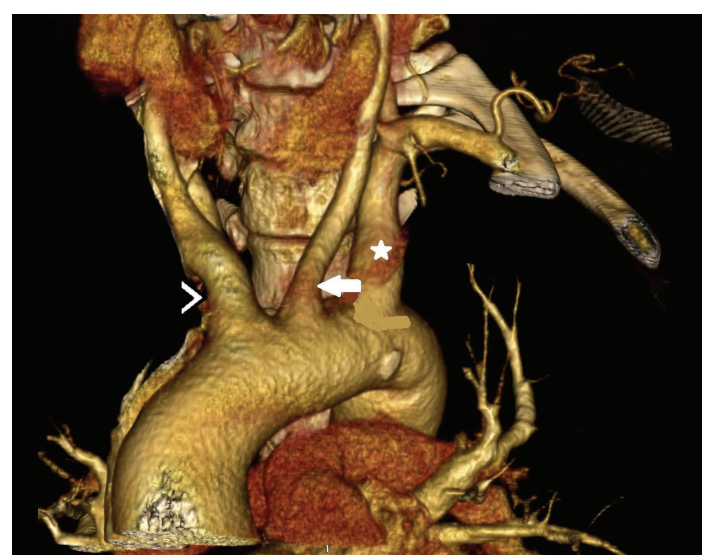

FIGURE 1: Three-dimensional volume rendered image shows brachiocephalic trunk (white arrow head), left common carotid artery (white arrow), and left subclavian artery (white star).

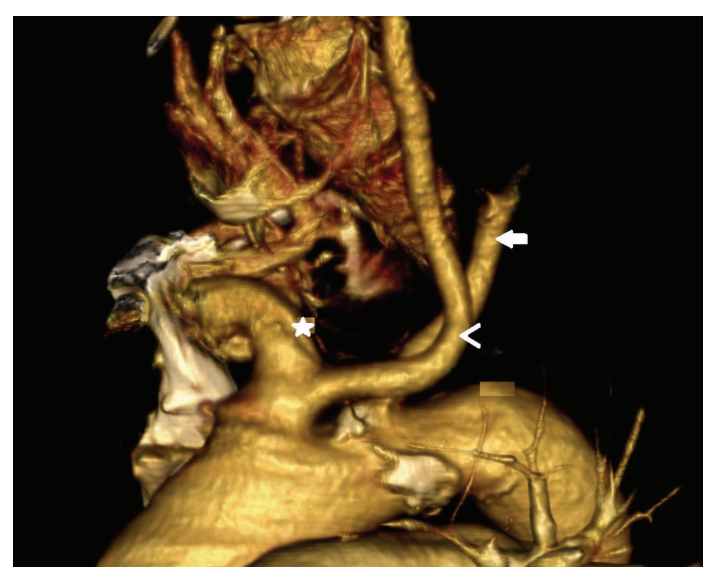

FIGURE 2: Three-dimensional volume rendered image demonstrates the left common carotid artery (white arrow head) originating from brachiocephalic trunk (white star) (white arrow head, left subclavian artery).

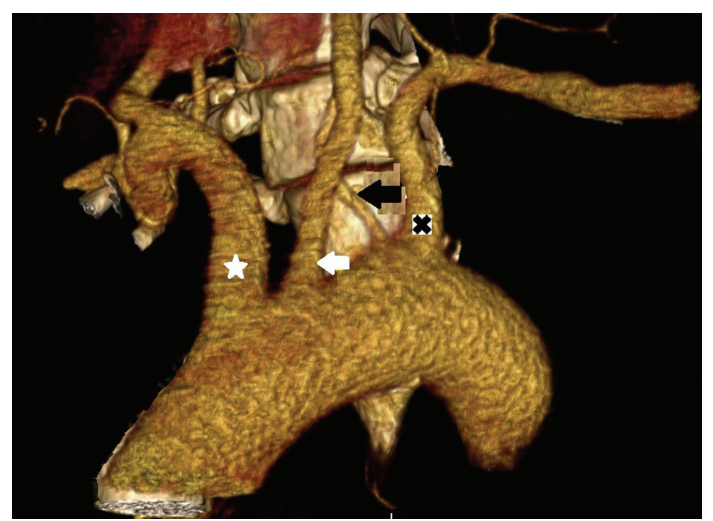

FIGURE 3: Three-dimensional volume rendered image shows the left vertebral artery (black arrow) originating from aortic arch between the left common carotid artery (white arrow) and left subclavian artery (black cross) (white star, brachiocephalic trunk). 


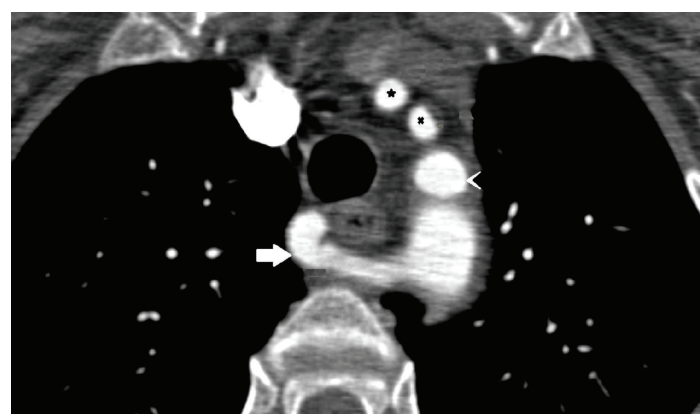

FIGURE 4: Axial maximum intensity projection image demonstrates the aberrant right subclavian artery (white arrow) originating from the last branch of the aortic arch and coursing to the right behind the esophagus (black star, right common carotid artery; black cross, left common carotid artery; white arrow head, left subclavian artery).

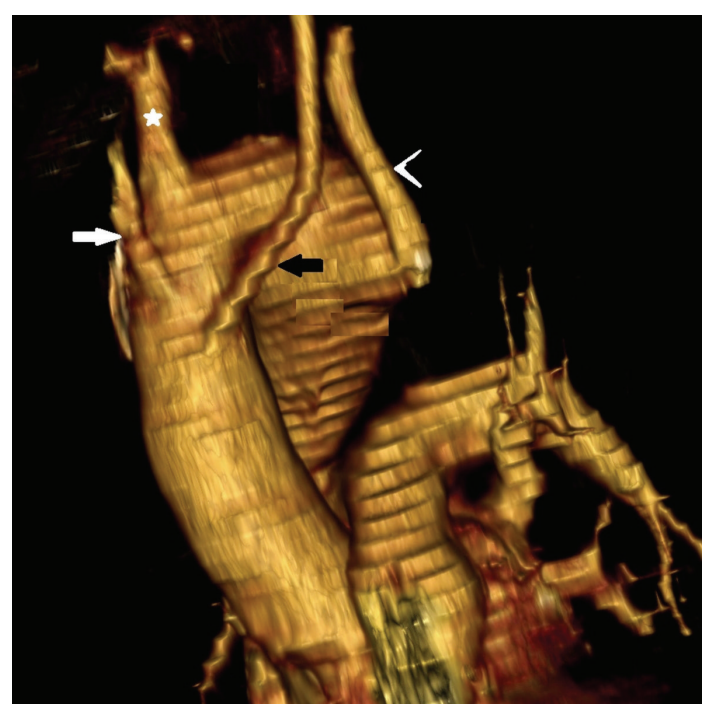

FIGURE 5: Three-dimensional volume rendered image shows the right aortic arch with aberrant left subclavian artery (black arrow) (white star, right common carotid artery; white arrow, right subclavian artery; white arrow head, left common carotid artery).

3 (1 male, 2 females) cases showed right aortic arch. Two cases had right aortic arch with aberrant left subclavian artery. The vessels originated in the following order: LCCA, RCCA, right SCA, and left SCA (Figure 5). One case with right aortic arch had mirror image type (left BCT, RCCA, and right SCA) (Figures 6 and 7).

In one female patient $(0.1 \%)$, the right vertebral artery (RVA) originated from the AA as terminal branch. It passed behind the esophagus and trachea and entered transvers foramina at the level of the seventh cervical vertebra. BCT with LCCA was also present in the same patient. The order of the branches was BCT with LCCA, left SCA, and RVA (Figures 8 and 9).

Double aortic arch was observed in one male patient (0.1\%). The ascending aorta divided into two arches that passed to either side of the esophagus and trachea and reunited to form the descending aorta (Figure 10).

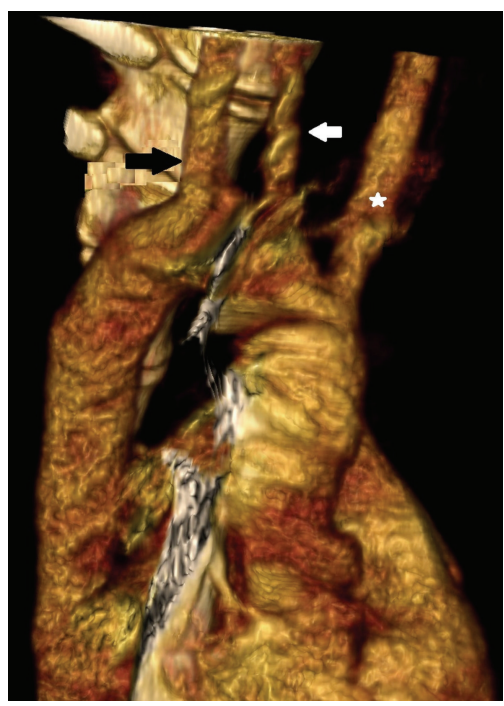

FIGURE 6: Three-dimensional volume rendered image demonstrates the right aortic arch with mirror-image type (black arrow, right subclavian artery; white arrow, right common carotid artery; white star, left brachiocephalic trunk).

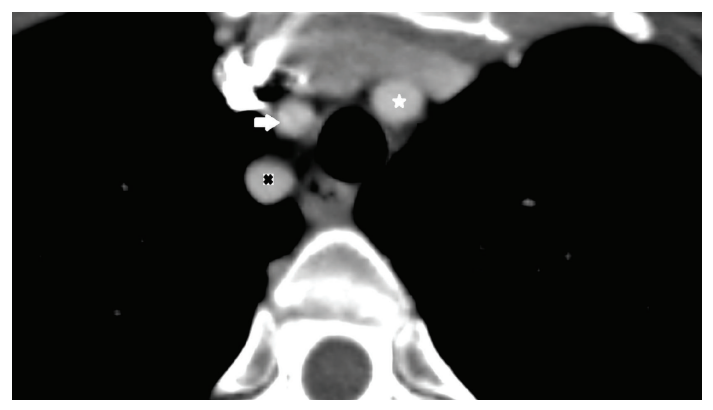

FIGURE 7: Axial image of the same patient with mirror-image type right aortic arch (white star, left brachiocephalic trunk; white arrow, right common carotid artery; black cross, right subclavian artery).

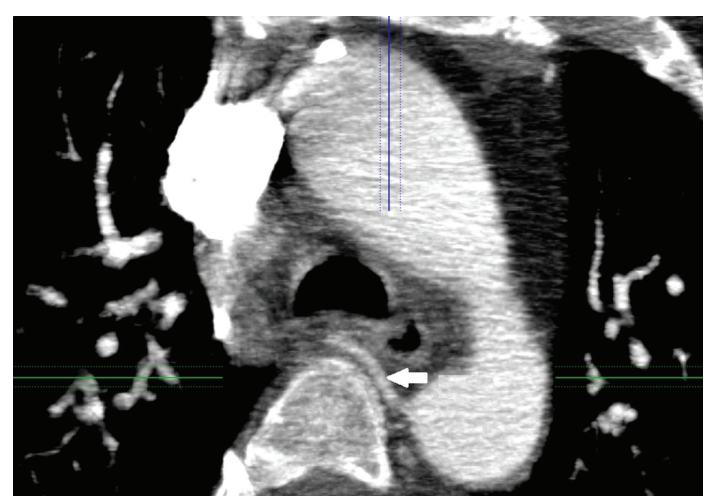

FIGURE 8: Axial maximum intensity projection image demonstrates the right vertebral artery (white arrow) originating from the aortic arch as the last branch. 


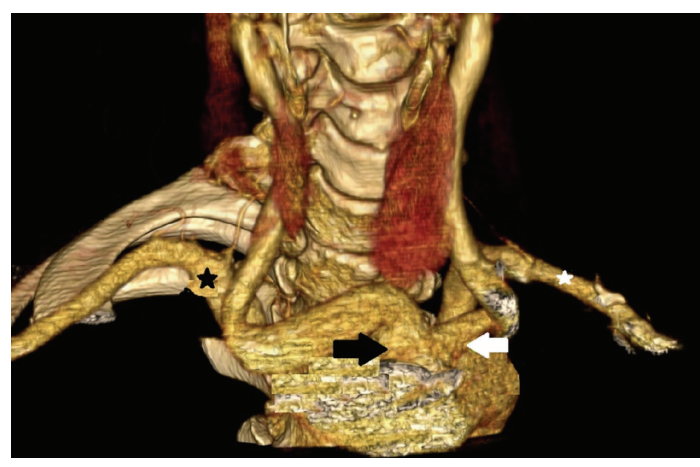

FIGURE 9: Three-dimensional volume rendered image of the same patient with right vertebral artery originating from aortic arch with left common carotid artery (white arrow) originating from brachiocephalic trunk (black arrow) (black star, right subclavian artery; white star, left subclavian artery).

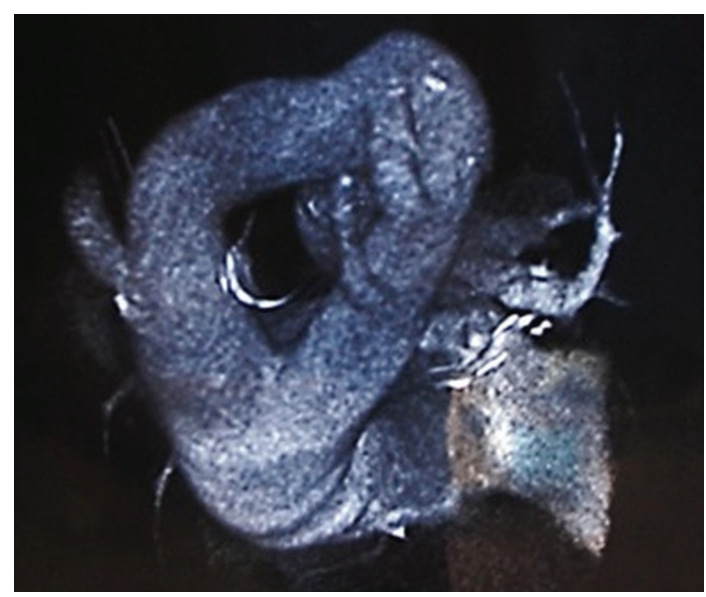

FIGURE 10: Three-dimensional volume rendered image demonstrates the double aortic arch.

\section{Discussion}

In the present study the normal three-branch pattern was observed in $87.4 \%$ of the cases; however, in $24.9 \%$ cases, the AA showed variations from usual branching pattern. The normal three-branch pattern of the AA is found with an incidence of 64.9-94.3\% according to the literature [1-6]. Our relative incidence of $87.4 \%$ lies in this wide range.

The most common variation of the AA with two branches (BCT with LCCA and left SCA) is found with an incidence of $10-22 \%$ in the literature $[1,7-11]$. This type is also called "bovine aortic arch" [7, 12, 13]. However, it is a misnomer because it does not in fact resemble the AA of the cattle which has only one branch that branches into right subclavian artery, a common truncus for common carotid arteries and left subclavian artery [7]. Clinical symptoms related to this variation have been reported and attributed to the widening of the mediastinum [14]. Developmentally, the two-branch pattern of the AA may be explained as follows: aortic sac normally bifurcates into right and left limbs. Left limb of the aortic sac forms the part of arch that intervenes between the origins of BCT and LCCA. If the aortic sac fails to bifurcate, BCT and LCCA will connect to aortic sac directly resulting in bifurcated trunk or common trunk giving origin to LCCA $[6,11]$.

Origination of the left vertebral artery from the aortic arch is not uncommon and the reported prevalence is between 2.4 and $8 \%[8,15]$. The most frequent location is between the LCCA and left SCA [5]. Occasionally, the left vertebral artery is the last branch of the AA. A case with anomalous origin of both vertebral arteries as additional branches of the AA distal to the left SCA has been reported [16]. In the current study, the left vertebral artery originated directly from AA between the LCCA and left SCA was observed in $2.8 \%$ of the cases. Normally the first part of vertebral artery develops from the dorsal ramus of the seventh intersegmental artery. In cases where vertebral artery arises as a branch from AA, embryologically it is explained that this is due to the fact that vertebral artery develops from the persistent sixth cervical intersegmental artery and segment of dorsal aorta fails to disappear, so blood flows through these persisting routes [6]. It is hypothesized that anomalous origins of the vertebral arteries lead to altered hemodynamics and predispose the patient to the formation of intracranial aneurysms. Satti et al. claim that patients with such anomalies should therefore be screened for coexisting aneurysms [17]. However, within the current literature, there is no conclusive evidence to suggest that anomalous origin of the vertebral arteries predisposes an individual to cerebrovascular disorders [18]. Among our cases with left vertebral artery originating from the aortic arch, we observed only one case with aneurysm of the clinoid segment of the right internal carotid artery.

Right aortic arch is an uncommon anatomical anomaly that occurs in $<0.1 \%$ of the population [19]. It results from the persistence of the right fourth branchial arch [5]. The most common type is right aortic arch with an aberrant left SCA. This type is rarely associated with congenital heart disease. Right aortic arch with mirror-image type is almost always associated with congenital heart disease, especially the cyanotic type [20]. In the current study two cases showed right aortic arch with aberrant left SCA type and one case showed right aortic arch with mirror-image type.

Double aortic arch is a rare anomaly caused by persistence of the fetal double aortic system [5]. It is a form of complete vascular ring resulting in noncardiac morbidity, but rarely associated with intracardiac defects [21]. The left carotid and subclavian arteries branch from the left arch, and the right carotid and subclavian arteries branch from the right arch. The right arch typically passes behind the esophagus, joins the left arch, and forms the aorta coursing down on the left. It forms a double aortic arch ring along with the ascending aorta and may compress the trachea and the esophagus. Symptoms typically present at an early age and are caused by compression of the trachea or esophagus [22]. The descending aorta is usually on the left side. Most commonly, one arch is dominant [5]. The right dominant arch is seen approximately in 70-80\%, codominant arch is seen in 5\%, and left dominant arch is seen in $25 \%$. In the current study, one case showed double aortic arch with nearly the same caliber (codominant arch). 
The right subclavian artery is the last branch of the AA in approximately $1 \%$ of individuals [23]. In the literature, the percentage range is between $0.13 \%$ and $25 \%$. [8]. Right SCA courses to the right behind the esophagus in $80 \%$ of the cases, between the esophagus and trachea in $15 \%$, and anterior to the trachea or mainstem bronchus in 5\% [5]. This variation, which is also called "arteria lusoria," is one of the most common vascular ring variations of the AA. Retroesophageal course may cause dysphagia [1]. Other variations of the AA may accompany the right aberrant subclavian artery. The most frequent is a common trunk for carotid arteries which accounts for 20.6-29\% of the right retroesophageal subclavian arteries [24]. We observed 17 cases $(1.9 \%)$ with aberrant right SCA and none of them has associated variations of the AA. All of the cases were asymptomatic and the aberrant right SCA was observed incidentally. In all of the cases the aberrant right SCA passed behind the esophagus.

One case showed LCCA originating from the BCT and right vertebral artery originating from the $\mathrm{AA}$ as the last branch distal to the origin of the left SCA. In the literature, few cases in which right vertebral artery originated directly from aortic arch have been reported $[20,25,26]$.

Natsis et al. investigated the aortic arches of 633 patients who had undergone digital subtraction angiography and determined 8 types of aortic arches [24]. Type I is called "normal" aortic arch branching into brachiocephalic trunk, left common carotid artery, and left subclavian artery. In type II, left common carotid artery originates from the brachiocephalic trunk. In type III, left vertebral artery directly leaves the aortic arch between the left common carotid artery and subclavian artery. In type IV, common carotid arteries originate from common trunk. In this type, the order of vessels from right to left is right subclavian artery, right and left common carotid arteries leaving from a common trunk, and left subclavian artery. In type V, common carotid arteries originate from a common trunk and a right aberrant subclavian artery is present. In type VI, common carotid arteries originate from a common trunk, subclavian arteries also originate from a common trunk. Brachiocephalic trunk is absent in type VII, and right subclavian artery, right common carotid artery, left common carotid artery, and left subclavian artery leave the aortic arch separately. In type VIII, thyroid ima artery originates from the aortic arch. In our study, patients with types I, II, and III only were present, and no other types were seen. In cases with right aberrant subclavian artery, right and left common carotid arteries left the aortic arch separately, which is a different finding from Natsis et al's study. Also in our study, double aortic arch, right aortic arch, and right vertebral artery originated from the aortic arc as a last branch. Natsis et al.s study did not include this classification.

Variations in the branching pattern of the AA are not rare. Most of them are asymptomatic and mostly found as an incidental finding during routine diagnostic procedures. However, head and neck surgeons and interventional radiologists should be aware of aortic arch variations. Identification of these vascular variations before diagnostic interventions is crucial in order to avoid complications. CTA is a reliable imaging method for demonstrating anatomical features and variations of the AA.

\section{Conflict of Interests}

The authors declare that there is no conflict of interests regarding the publication of this paper.

\section{References}

[1] T. Kau, M. Sinzig, J. Gasser et al., "Aortic development and anomalies," Seminars in Interventional Radiology, vol. 24, no. 2, pp. 141-152, 2007.

[2] H. A. Alsaif and W. S. Ramadan, "An anatomical study of the aortic arch anomalies," Journal of King Abdulaziz UniversityMedical Sciences, vol. 17, pp. 37-54, 2010.

[3] G. C. Jakanani and W. Adair, "Frequency of variations in aortic arch anatomy depicted on multidetector CT," Clinical Radiology, vol. 65, no. 6, pp. 481-487, 2010.

[4] M. Müller, B. L. Schmitz, S. Pauls et al., "Variations of the aortic arch-a study on the most common branching patterns," Acta Radiologica, vol. 52, no. 7, pp. 738-742, 2011.

[5] S. Kadir, "Regional anatomy of the thoracic aorta," in Atlas of Normal and Variant Angiographic Anatomy, S. Kadir, Ed., WB Saunders, Philadelphia, Pa, USA, 1991.

[6] S. R. Nayak, M. M. Pai, L. V. Prabhu, S. D'Costa, and P. Shetty, "Anatomical organization of aortic arch variations in the India: embryological basis and review," Jornal Vascular Brasileiro, vol. 5, no. 2, pp. 95-100, 2006.

[7] K. F. Layton, D. F. Kallmes, H. J. Cloft, E. P. Lindell, and V. S. Cox, "Bovine aortic arch variant in humans: clarification of a common misnomer," American Journal of Neuroradiology, vol. 27, no. 7, pp. 1541-1542, 2006.

[8] R. A. Bergman, A. K. Afifi, and R. Miyauchi, "Illustrated encyclopedia of human anatomic variation," 2000, http://www.anatomyatlases.org/AnatomicVariants/Cardiovascular/Text/Arteries/Aorta.shtml.

[9] N. R. Grande, A. Costa e Silva, A. S. Pereira, and A. P. Aguas, "Variations in the anatomical organization of the human aortic arch. A study in a Portuguese population," Bulletin de l'Association des Anatomistes, vol. 79, no. 244, pp. 19-22, 1995.

[10] M. L. Nelson and C. D. Sparks, "Unusual aortic arch variations: distal origin of common carotid arteries," Clinical Anatomy, vol. 14, no. 1, pp. 62-65, 2001.

[11] G. A. Poultsides, E. D. Lolis, J. Vasquez, A. D. Drezner, and D. Venieratos, "Common origins of carotid and subclavian arterial systems: report of a rare aortic arch variant," Annals of Vascular Surgery, vol. 18, no. 5, pp. 597-600, 2004.

[12] A. Azakie, D. B. McElhinney, L. M. Messina, and R. J. Stoney, "Common brachiocephalic trunk: Strategies for revascularization," Annals of Thoracic Surgery, vol. 67, no. 3, pp. 657-660, 1999.

[13] L. J. Lamers, D. G. Rowland, J. H. Seguin, E. M. Rosenberg, and K. M. Reber, "The effect of common origin of the carotid arteries in neurologic outcome after neonatal ECMO," Journal of Pediatric Surgery, vol. 39, no. 4, pp. 532-536, 2004.

[14] K. P. Karkoulias, G. K. Efremidis, M. S. Tsiamita et al., "Abnormal origin of the left common carotid artery by innominate artery: a case of enlargement mediastinum," Monaldi Archives for Chest Disease, vol. 59, no. 3, pp. 222-223, 2003. 
[15] J. D. Liechty, T. W. Shields, and B. J. Anson, "Variations pertaining to the aortic arches and their branches," Quarterly Bulletin of Northwestern University Medical School, vol. 31, no. 2, pp. 136-143, 1957.

[16] V. B. Goray, A. R. Joshi, A. Garg, S. Merchant, B. Yadav, and P. Maheshwari, "Aortic arch variation: a unique case with anomalous origin of both vertebral arteries as additional branches of the aortic arch distal to left subclavian artery," American Journal of Neuroradiology, vol. 26, no. 1, pp. 93-95, 2005.

[17] S. R. Satti, C. A. Cerniglia, and R. A. Koenigsberg, "Cervical vertebral artery variations: an anatomic study," American Journal of Neuroradiology, vol. 28, no. 5, pp. 976-980, 2007.

[18] S. Albayram, P. Gailloud, and B. A. Wasserman, "Bilateral arch origin of the vertebral arteries," American Journal of Neuroradiology, vol. 23, no. 3, pp. 455-458, 2002.

[19] C. S. Cina, H. Althani, J. Pasanau, and L. Abouzahr, "Kommerel's diverticulum and right sided aortic arch: a coherty study and review of the literatüre," Journal of Vascular Surgery, vol. 39, pp. 131-139, 2004.

[20] D. B. McElhinney, A. K. Hoydu, J. W. Gaynor, T. L. Spray, E. Goldmuntz, and P. M. Weinberg, "Patterns of right aortic arch and mirror-image branching of the brachiocephalic vessels without associated anomalies," Pediatric Cardiology, vol. 22, no. 4, pp. 285-291, 2001.

[21] K. Alsenaidi, R. Gurofskya, T. Karamlou, W. G. Williams, and B. W. McCrindle, "Management and outcomes of double aortic arch in 81 patients," Pediatrics, vol. 118, no. 5, pp. el336-e1341, 2006.

[22] F. Dicenta, J. A. Rodríguez, and V. Belloch, "Double aortic arch," Revista Espanola de Cardiologia, vol. 55, no. 10, p. 1088, 2002.

[23] J. V. Richardson, D. B. Doty, N. P. Rossi, and J. L. Ehrenhaft, "Operation for aortic arch anomalies," Annals of Thoracic Surgery, vol. 31, no. 5, pp. 426-432, 1981.

[24] K. I. Natsis, I. A. Tsitouridis, M. V. Didagelos, A. A. Fillipidis, K. G. Vlasis, and P. D. Tsikaras, "Anatomical variations in the branches of the human aortic arch in 633 angiographies: clinical significance and literature review," Surgical and Radiologic Anatomy, vol. 31, no. 5, pp. 319-323, 2009.

[25] I. M. Best and H. L. Bumpers, "Anomalous origins of the right vertebral, subclavian, and common carotid arteries in a patient with a four-vessel aortic arch," Annals of Vascular Surgery, vol. 16, no. 2, pp. 231-234, 2002.

[26] M. Karcaaltincaba, J. Strottman, and L. Washington, "Multidedector-row CT angiography findings in the bilateral aortic arch origin of the vertebral arteries," American Journal of Neuroradiology, vol. 24, no. 1, article 157, 2003. 


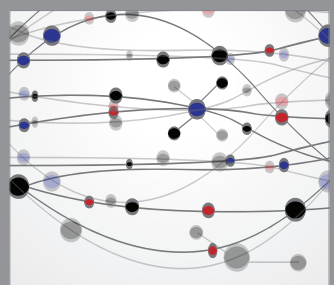

The Scientific World Journal
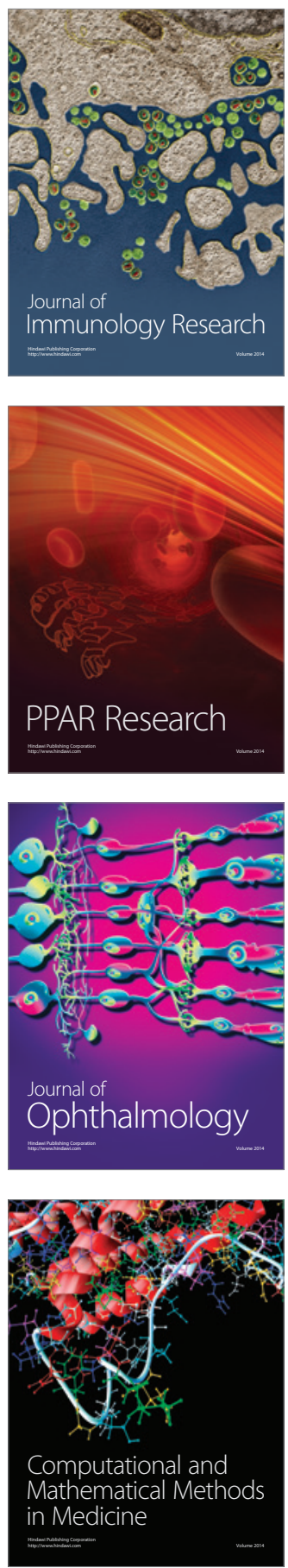

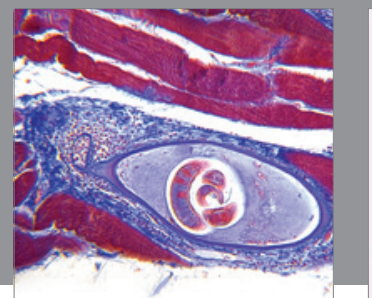

Gastroenterology

Research and Practice
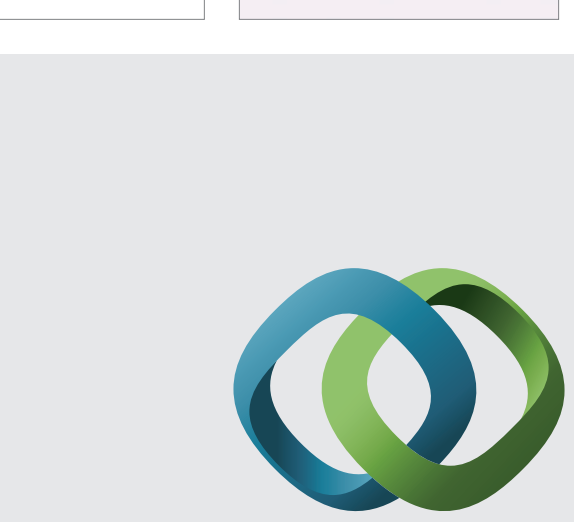

\section{Hindawi}

Submit your manuscripts at

http://www.hindawi.com
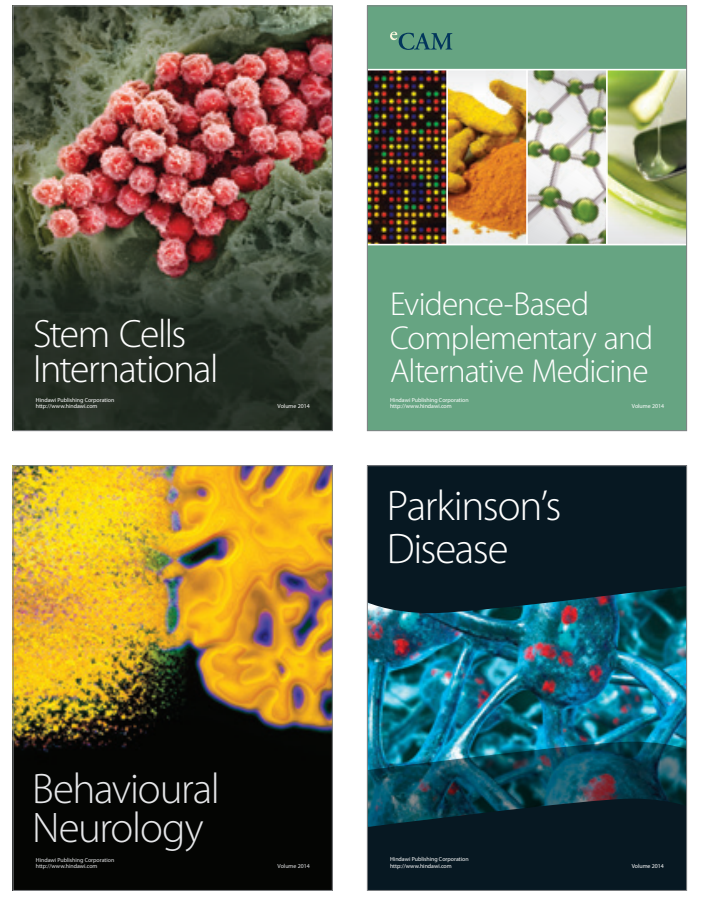
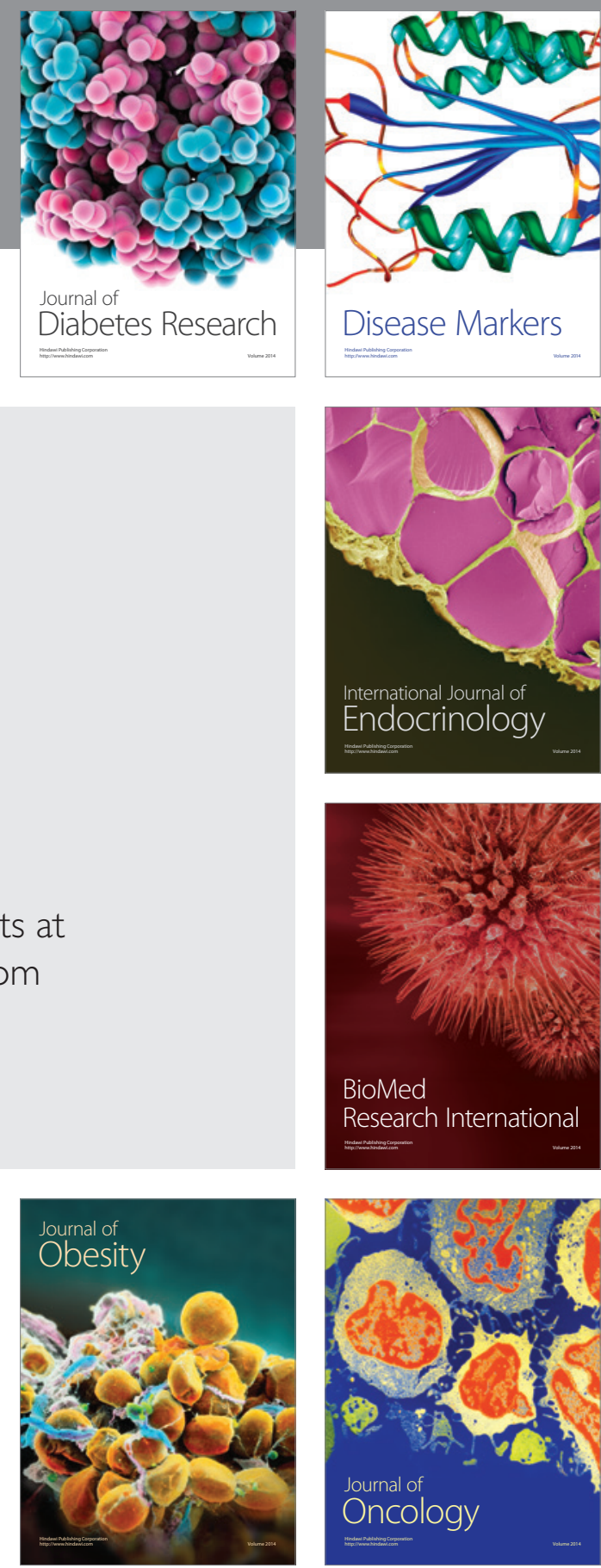

Disease Markers
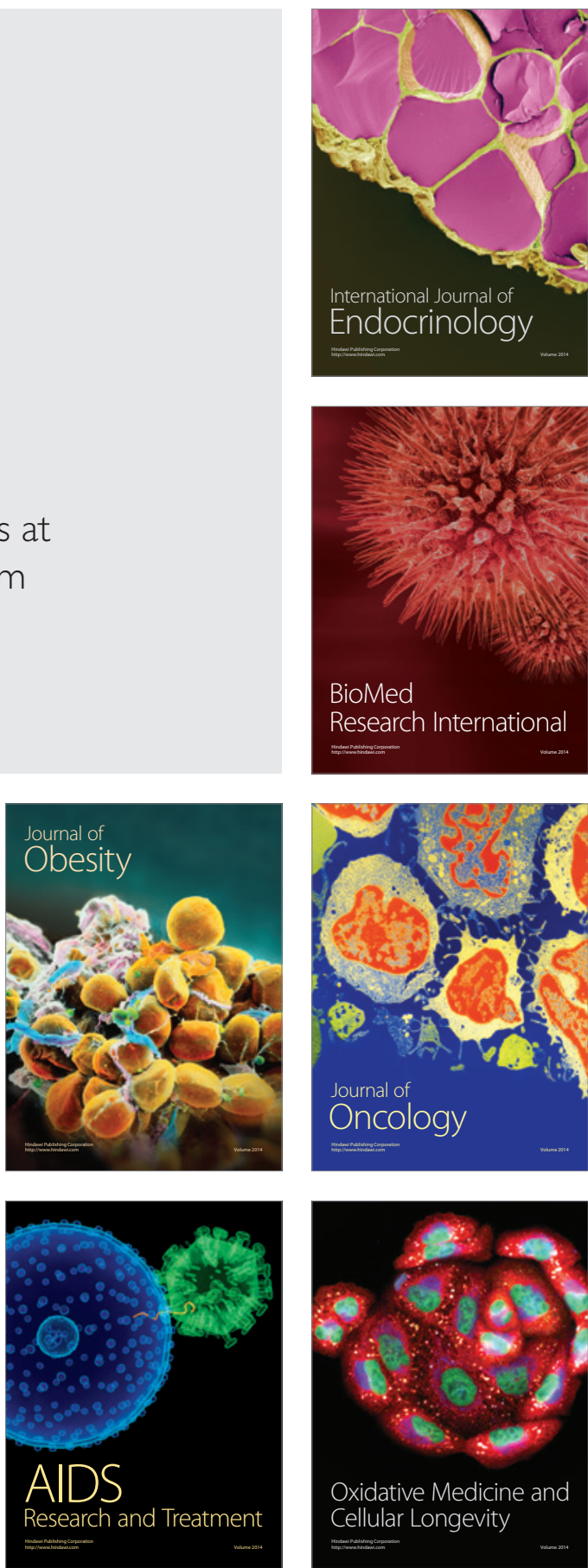